\title{
Level of accumulation of epoxy fatty acid in Arabidopsis thaliana expressing a linoleic acid $\Delta 12$-epoxygenase is influenced by the availability of the substrate linoleic acid
}

Received: 31 March 2004 / Accepted: 5 May 2004 / Published online: 24 June 2004

(C) Springer-Verlag 2004

\begin{abstract}
Arabidopsis thaliana (L.) Heynh. expressing the Crepis palaestina (L.) linoleic acid $\Delta 12$-epoxygenase in its developing seeds typically accumulates low levels of vernolic acid (12,13-epoxy-octadec-cis-9-enoic acid) in comparison to levels found in seeds of the native $C$. palaestina. In order to determine some of the factors limiting the accumulation of this unusual fatty acid, we have examined the effects of increasing the availability of linoleic acid (9cis, 12cis-octadecadienoic acid), the substrate of the $\Delta 12$-epoxygenase, on the quantity of epoxy fatty acids accumulating in transgenic A. thaliana. The addition of linoleic acid to liquid cultures of transgenic plants expressing the $\Delta 12$-epoxygenase under the control of the cauliflower mosaic virus $35 \mathrm{~S}$ promoter increased the amount of vernolic acid in vegetative tissues by 2.8 -fold. In contrast, the addition to these cultures of linoelaidic acid (9trans, 12trans-octadecadienoic acid), which is not a substrate of the $\Delta 12$-epoxygenase, resulted in a slight decrease in vernolic acid accumulation. Expression of the $\Delta 12$-epoxygenase under the control of the napin promoter in the A. thaliana triple mutant $\mathrm{fad} 3 / \mathrm{fad} 7-1 / \mathrm{fad} 8$, which is deficient in the synthesis of tri-unsaturated fatty acids and has a $60 \%$ higher level of linoleic acid than the wild type, was found to increase the average vernolic acid content of the seeds by $55 \%$ compared to the expression of the $\Delta 12$ epoxygenase in a wild-type background. Together, these
\end{abstract}

Communicated by L. Willmitzer

E. Rezzonico $\cdot$ L. Moire $\cdot$ S. Delessert $\cdot$ Y. Poirier $(\bowtie)$ Département de Biologie Moléculaire Végétale, Université de Lausanne,

1015 Lausanne, Switzerland

e-mail: yves.poirier@ie-bpv.unil.ch

Tel.: +41-21-6924222

Fax: +41-21-6924195

Present address:

E. Rezzonico

Nutrition and Health Department, Functional Microbiology

Group, Nestlé Research Center,

P.O. Box 44, 1000 Lausanne 26, Switzerland results reveal that the availability of linoleic acid is an important factor affecting the synthesis of epoxy fatty acid in transgenic plants.

\section{Introduction}

While the membranes of plant cells are composed primarily of five "common" fatty acids - namely, stearic, palmitic, oleic, linoleic and linolenic acids - a very large diversity of fatty acids exists in the reserve triacylglycerols (TAG) of seeds, and nearly 300 naturally occurring fatty acids have been described there to date (Badami and Patil 1981; van de Loo et al. 1993). The structures of these latter fatty acids vary in a number of features, including the length of the acyl chains, the number, position and nature of the unsaturated bonds and the presence of functional groups, such as the hydroxy, epoxy and acetylenic groups. They are collectively referred as "unusual" fatty acids since their structures are different from those of the common fatty acids found in membranes.

Several genes have recently been identified that code for enzymes involved in the synthesis of fatty acids containing unusual groups (for a recent review see Jaworski and Cahoon 2003). In the majority of cases, these enzymes were found to be variants of enzymes involved in the synthesis of the common fatty acids, such as variants of the oleic acid $\Delta 12$-desaturase (FAD2) or of the soluble stearoyl-acyl carrier protein (ACP) desaturase (Cahoon et al. 1992; van de Loo et al. 1995). Novel enzymes have also been identified that are thought to play an important role in directing unusual fatty acids towards the storage TAG and away from membrane lipids (Dahlqvist et al. 2000).

Many of the unusual fatty acids have properties that are valuable as renewable feedstocks for the chemical industry. For example, vernolic acid (12,13-epoxy-octadec-cis-9-enoic acid) can be used as a plasticizer of polyvinyl chloride, as a precursor in the manufacture of nylon or as a component of adhesives and paints (Budziszewski et al. 1996). Although several native 
plants, such as Crepis palaestina or Euphorbia lagascae, naturally accumulate between $50 \%$ and $90 \%$ of their seed fatty acids as vernolic acid, they have undesired agronomical features which make them unsuitable as agricultural crops. Efforts have thus been made to transfer the biosynthetic pathway for vernolic acid, as well as for other unusual fatty acids, into present-day oil crop species, such as rapeseed and the model oilseed plant Arabidopsis thaliana. However, in contrast to the native plants, the majority of transgenic plants expressing genes involved in the synthesis of unusual fatty acids, including vernolic acid, accumulate only low amounts (less than $20 \%$ ) of these fatty acids in their seed oil (Jaworski and Cahoon 2003; Voelker and Kinney 2001). Further progress will depend on identifying the factors limiting the accumulation of unusual fatty acids in oilseed crops and on coordinating the expression of several transgenes capable of correcting these limitations.

The accumulation of unusual fatty acid in TAG can be divided into two major steps: first, the insertion of the functional group into the acyl chain and second, the stable incorporation of the unusual fatty acids into TAG. Failure to accumulate unusual fatty acids into oil seeds of transgenic plants could be caused by a number of factors, including low gene expression, poor activity of the fatty acid-modifying enzyme, inappropriate amount or access of the substrate or co-factors to the enzyme or preferential degradation of the newly synthesized unusual fatty acid.

The purpose of the investigation reported here was to analyze the potential role of substrate limitation on the synthesis of vernolic in transgenic $A$. thaliana expressing the $C$. palaestina linoleic acid $\Delta 12$-epoxygenase in developing seeds. The $C$. palaestina $\Delta 12$-epoxygenase is a desaturase-type enzyme that converts the $\Delta 12$ double bond of linoleic acid into an epoxy group (Lee et al. 1998). We performed experiments to examine how modulation of the quantity of linoleic acid influences the synthesis of vernolic acid in transgenic plants.

\section{Materials and methods}

DNA constructs

The Crepis palaestina linoleic acid $\Delta 12$-epoxygenase cDNA (Y16283) (kindly provided by S. Stymne, Swedish University of Agricultural Sciences, Alnarp, Sweden) was cloned as a BamHI fragment either in the pART7 vector, thereby placing the gene under the control of the cauliflower mosaic virus (CaMV) 35S promoter, or in the variant pART7-napin vector, putting the gene under the control of the 1.1-kbp Brassica napus napin promoter (Gleave 1992; Poirier et al. 1999). The cDNA with the upstream and downstream regulatory elements were then sub-cloned as a SacI-PstI fragment in the pCAMBIA 1300 vector (http://www.cambia.org.au) containing the hygromycin resistance gene under the control of CaMV $35 \mathrm{~S}$ promoter. All binary vectors were transferred into Agrobacterium tumefaciens strain pGV3101 by electroporation.
Bacterial and plant material

Wild-type Arabidopsis thaliana, the fad3/fad7-1/fad8 mutant (McConn and Browse 1996) and the transgenic A. thaliana line PHA10.3 (formerly described as N-PHA-10.3) expressing the Pseudomonas aeruginosa PHAC1 synthase modified for peroxisome targeting under the control of the 1.1-kbp Brassica napus napin promoter (Poirier et al. 1999), were transformed with the pCAMBIA 1300 vector or constructs containing the $\Delta 12$-epoxygenase gene by the floral dip method (Clough and Bent 1998). $\mathrm{T}_{1}$ transformants were isolated by plating seeds on medium containing half-strength MS salts (Murashige and Skoog 1962), 1\% (w/v) sucrose, $0.7 \%(\mathrm{w} / \mathrm{v})$ agar and $30 \mu \mathrm{g} / \mathrm{ml}$ hygromycin. Hygromycinresistant plants were subsequently transferred to soil and grown under continuous fluorescent light at $19^{\circ} \mathrm{C}$.

For feeding experiments, axenic plants were first grown under constant agitation $(100 \mathrm{rpm})$ for 9 days in liquid media containing half-strength MS salts and $2 \%$ sucrose before linoleic acid or linoelaidic acid was added at a concentration of $0.1 \%(\mathrm{v} / \mathrm{v})$ for $12 \mathrm{~h}$. The plants were then collected, washed extensively with water and freeze-dried. Linoleic acid and linoelaidic acid were purchased from Nu-Chek-Prep (Minnesota, USA).

\section{Fatty acid analysis}

Seed or whole-plant fatty acid methyl-esters were prepared by acidcatalysed $\left(1 \mathrm{~N}\right.$ HCL in methanol, $\left.2 \mathrm{~h}, 80^{\circ} \mathrm{C}\right)$ or base-catalysed $\left(0.1 \mathrm{M}\right.$ sodium methoxide in methanol, $\left.1 \mathrm{~h}, 60^{\circ} \mathrm{C}\right)$ trans-esterification. Following the reaction, the fatty acid methyl-esters were extracted with hexane and water and the organic phase transferred to vials. Analysis was performed using a gas chromatograph equipped with a glass capillary column (model SP230, Supelco, Bellefonte, $\mathrm{Pa}$.) and a flame ionization detector.

\section{Results and discussion}

External supply of linoleic acid to cultures of transgenic plants increases the amount of epoxy fatty acid in vegetative tissues

The presence of epoxy fatty acids in the lipids of transgenic $A$. thaliana expressing the $C$. palaestina $\Delta 12$ epoxygenase under the control of the constitutive CaMV 35S promoter is typically undetectable in adult rosettes of soil-grown plants. Similar results have been observed for transgenic plants expressing either the California bay lauroyl-ACP thioesterase or the Ricinus communis oleic acid $\Delta 12$-hydroxylase in vegetative tissues (Broun et al. 1998; Eccleston et al. 1996). In contrast, epoxy fatty acids are detectable in transgenic lines expressing the $C$. palaestina $\Delta 12$-epoxygenase under control of the CaMV 35S promoter when the lipids of 8- to 10-day-old seedlings grown in liquid culture containing sucrose are examined (data not shown). Consequently we used just such a transgenic line (WTSE2.6), which contained approximately $2.8 \mathrm{~mol} \%$ vernolic acid and $1.8 \mathrm{~mol} \%$ 12,13-epoxy-octadeca-9cis, $15 \mathrm{cis}$-dienoic acid (hereafter referred as 12-epoxy-18:2) in its lipid fraction when grown for 9 days in liquid culture, as a first approach to examine the effect of linoleic acid availability on the accumulation of epoxy fatty acids. A 
transgenic line transformed with the empty binary vector was used as a control (WTPC7.3).

External free or esterified fatty acids added to either cell cultures or plant tissues have been shown to be readily metabolized and incorporated into a wide range of lipids, including phospholipids, glycolipids and TAG (Stumpf and Weber 1977; Terzaghi 1986a, b, 1989). We have used a similar system to manipulate the level of linoleic acid in plant cells. Transgenic plants were grown for 9 days in media without external fatty acids, followed by the addition of either linoleic acid or linoelaidic acid (9trans, 12trans-octadecadienoic acid) at a final concentration of $0.1 \%(\mathrm{v} / \mathrm{v})$ for $12 \mathrm{~h}$. Linoelaidic acid was used as a control because this fatty acid is not a substrate for the $\Delta 12$-epoxygenase and cannot be converted to linoleic acid. Since the external linoleic and linoelaidic acid cannot be effectively removed from the plant material before fatty acid analysis (data not shown), the proportion of epoxy fatty acids was expressed as a fraction of the total fatty acids composed of palmitic acid, oleic acid, linolenic acid, vernolic acid and 12-epoxy-18:2 (Table 1).

Addition of linoleic acid was found to increase the level of vernolic acid in line WTSE2.6 from $3.2 \mathrm{~mol} \%$ to $7.4 \mathrm{~mol} \%$, while the amount of 12-epoxy-18:2 increased from $1.8 \mathrm{~mol} \%$ to $2.3 \mathrm{~mol} \%$. In contrast, addition of linoelaidic acid had little impact on the amount epoxy fatty acids, with vernolic acid decreasing slightly but significantly from $3.2 \mathrm{~mol} \%$ to $2.5 \mathrm{~mol} \%$ while 12 -epoxy- $18: 2$ remained unchanged at $1.7-1.8 \mathrm{~mol} \%$. In addition to the synthesis of epoxy fatty acid, expression of the $\Delta 12$ epoxygenase in line WTSE2.6 resulted in an approximate twofold increase in the proportion of oleic acid under all treatments. Similar changes in oleic acid contents have been previously observed in transgenic plants producing vernolic acid as a result of the expression of the $C$. palaestina $\Delta 12$-epoxygenase or the E. lagascae cytochrome P450 epoxygenase as well as in plants expressing other types of divergent oleic acid $\Delta 12$-desaturases involved in the synthesis of hydroxylated or conjugated unsaturated fatty acid (Broun and Somerville 1997; Broun et al. 1998; Cahoon et al. 1999, 2002; Singh et al. 2001; Smith et al. 2003). The increase in the proportion of oleic acid resulting from the expression of the epoxygenase was found to be unaffected by the addition of either linoleic acid or linoelaidic acid. Addition of either linoleic or linoelaidic acid also did not lead to significant changes in the proportion of palmitic, oleic and linolenic acid in the control line WTPC7.3. Together, these data indicate that the increase in epoxy fatty acid observed after the addition of linoleic acid to cultures of plants expressing the $\Delta 12$ epoxygenase is specific for this fatty acid and that the addition of fatty acids to the growth media for $12 \mathrm{~h}$ does not result in an unspecific perturbation of the overall profile of common fatty acids.

Accumulation of epoxy fatty acids in seeds of mutant plants with an increased level of linoleic acid

Genetic engineering of plants aimed at the production of unusual fatty acids primarily targets manipulation of the lipid biosynthetic pathway in developing seeds. The possibility that the availability of linoleic acid may be also influencing the synthesis of epoxy fatty acids in developing seeds was thus examined in transgenic plants expressing the $C$. palaestina $\Delta 12$-epoxygenase under the control of the $B$. napus napin promoter. The $A$. thaliana triple mutant $\mathrm{fad} 3 / \mathrm{fad} 7-1 / \mathrm{fad} 8$ has been shown to be strongly deficient in linoleic acid desaturase activity, leading to a strong reduction in the accumulation of trienoic acids and a significant increase in dienoic acids in leaves (McConn and Browse 1996). Analysis of the fatty acid profile in mature seeds revealed a $60 \%$ increase of the proportion of linoleic acid, from $29 \mathrm{~mol} \%$ in the wild type up to $46 \mathrm{~mol} \%$ in the triple mutant (Table 2). The amount of epoxy fatty acid (vernolic acid +12 -epoxy-18:2) was determined for seeds of plants obtained from the transformation of the same napin-epoxygenase construct into three different lines, namely the fad3/fad7-1/fad8 mutant, a transgenic line expressing a bacterial polyhydroxyalkanoate (PHA) synthase in the peroxisome and wild-type plants, all in the Columbia background. Expression of the bacterial PHA synthase in the peroxisome leads to polyhydroxyalkanoate synthesis from the intermediate of

Table 1 Effects of the addition of linoleic acid on the accumulation of epoxy fatty acids

\begin{tabular}{lllllll}
\hline Plant line & Treatment & \multicolumn{2}{l}{ Fatty acids $(\mathrm{mol} \%)^{\mathrm{a}}$} \\
\cline { 3 - 7 } & & $16: 0$ & $18: 1$ & $18: 3$ & Epoxy 1 & Epoxy 2 \\
\hline WTPC7.3 & No f.a. & $15 \pm 1$ & $4.0 \pm 0.2$ & $81 \pm 1$ & ND & ND \\
WTSE2.6 & No f.a. & $16 \pm 1$ & $8.7 \pm 0.2$ & $71 \pm 1$ & $3.2 \pm 0.5$ & $1.8 \pm 0.1$ \\
WTPC7.3 & $18: 2 \mathrm{c}$ & $15 \pm 1$ & $3.9 \pm 0.1$ & $81 \pm 1$ & ND & ND \\
WTSE2.6 & $18: 2 \mathrm{c}$ & $15 \pm 1$ & $8 \pm 1$ & $68 \pm 2$ & $7.4 \pm 0.4$ & $2.3 \pm 0.1$ \\
WTPC7.3 & $18: 2 \mathrm{t}$ & $14 \pm 1$ & $3.7 \pm 0.5$ & $82 \pm 1$ & ND & ND \\
WTSE2.6 & $18: 2 \mathrm{t}$ & $15 \pm 1$ & $7.3 \pm 0.8$ & $74 \pm 2$ & $2.5 \pm 0.2$ & $1.7 \pm 0.1$ \\
\hline
\end{tabular}

WTPC7.3 control plants transformned with the empty binary vector, WTSE2.6 transgenic plants expressing the $\Delta 12$-epoxygenase under the CaMV35S promoter

No f.a. no addition of fatty acids, $18: 2 c$ linoleic acid, $18: 2 t$ linoelaidic acid

$N D$ not detected

${ }^{\mathrm{a}}$ Values are derived from two independent experiments, mean $\pm \mathrm{SD}$ 
ß-oxidation and has previously been used to study the flux of fatty acids through the peroxisomal ß-oxidation cycle (Mittendorf et al. 1999; Poirier 2002). Plants expressing both the $C$. palaestina $\Delta 12$-epoxygenase and the peroxisomal PHA synthase in developing seeds have recently been used to reveal the presence of a futile cycle of epoxy fatty acid degradation through the peroxisomal ß-oxidation pathway (Moire et al. 2004). Since the expression of the PHA synthase in the peroxisome had no impact on seed fatty acid composition (data not shown), we regarded plants expressing both the PHA synthase and $\Delta 12$ epoxygenase as controls to the same extent as wild-type plants expressing only the $\Delta 12$-epoxygenase.

The amount of unusual fatty acids was determined for a pool of approximately 100 seeds harvested from each primary transgenic plants obtained from the transformation of the napin-epoxygenase construct into either wildtype (lines WTNE), the fad3/fad7-1/fad8 mutant (lines $f a d \mathrm{NE}$ ) or transgenic plants expressing the peroxisomal PHA synthase (lines PHANE) (Fig. 1). The amount of epoxy fatty acids present in mature seeds of 16 independent lines expressing the $\Delta 12$-epoxygenase in a wild-type background with and without the napin-PHA synthase varied between $0.5 \mathrm{~mol} \%$ and $2.9 \mathrm{~mol} \%$, (average $=2.2 \mathrm{~mol} \%$ ) with no statistically significant differences between the two groups. In contrast, expression of the napin-epoxygenase in the $\mathrm{fad} 3 / \mathrm{fad} 7-1 / \mathrm{fad} 8$ mutant led to a statistically significant increase in the amount of epoxy fatty acids in seeds, with a range of 2.3$4.6 \mathrm{~mol} \%$ (average $=3.4 \mathrm{~mol} \%$ ) epoxy fatty acids among 34 independent lines (Fig. 1). Comparison of the fatty acid profile in two fad3/fad7-1/fad 8 mutant lines ( $f a d \mathrm{NE} 1$ and fad $\mathrm{NE} 35)$ producing $2.9 \mathrm{~mol} \%$ and $3.5 \mathrm{~mol} \%$ vernolic acid, respectively, with the non-transgenic fad3/fad7-1/ fad 8 parental line revealed that expression of the $\Delta 12$ epoxygenase decreased the level of linoleic acid from
$46 \mathrm{~mol} \%$ to $19-24 \mathrm{~mol} \%$ and increased the level of oleic acid from $17 \mathrm{~mol} \%$ to $37-45 \mathrm{~mol} \%$. These levels of change in oleic and linoleic acid are similar to the ones observed when a vector-transformed line in a wild-type background (WTPC2.1) was compared with a line that expressed the $\Delta 12$-epoxygenase in the wild-type background and accumulated $2.1 \mathrm{~mol} \%$ epoxy fatty acids (line WTNE7.7) (Table 2). Furthermore, in contrast to the expression of the $\Delta 12$-epoxygenase in wild-type $A$. thaliana, expression of the $\Delta 12$-epoxygenase in the fad3/fad7-1/fad8 mutant led to the synthesis of only vernolic acid and not of 12-epoxy-18:2 (Table 2). These results are in accordance with the implication that omega-3 desaturases are involved in the accumulation of 12-epoxy18:2 either through the $\Delta 15$-desaturation of vernolic acid or the $\Delta 12$-epoxygenation of linolenic acid (Singh et al. 2001).

Levels of linoleic acid as an important factor for the synthesis of epoxy fatty acids in transgenic plants

Expression of the $C$. palaestina linoleic acid $\Delta 12$ epoxygenase in $A$ thaliana has been found to lead to a marked increase in oleic acid and decrease in linoleic and linolenic acids (Table 2) (Singh et al. 2001). Similar results have been obtained for transgenic plants expressing a $R$. communis or Lesquerella fendleri oleic acid $\Delta 12$-hydroxylase, or a Momordica charantia linoleic acid $\Delta 12$ conjugase, all members of the $\Delta 12$ oleic acid desaturase family, as well as in transgenic plants expressing a cytochrome $\mathrm{P} 450$ from E. lagascae involved in the synthesis of vernolic acid (Broun and Somerville 1997; Cahoon et al. 1999; Cahoon et al. 2002; Smith et al. 2003). One proposed hypothesis to explain this phenotype is that the accumulation of unusual fatty acids on phospholipids

Table 2 Fatty acid composition of mature seeds of transgenic lines expressing the epoxygenase gene

\begin{tabular}{|c|c|c|c|c|c|}
\hline \multirow[t]{2}{*}{ Fatty acids } & \multicolumn{5}{|c|}{ Fatty acid content ${ }^{\mathrm{a}}(\mathrm{mol} \%)$} \\
\hline & WTPC $2.1^{\mathrm{b}}$ & WTNE7. $7^{\mathrm{c}}$ & $\mathrm{fad} 3 / 7 / 8^{\mathrm{d}}$ & $f a d \mathrm{NE} 1^{\mathrm{e}}$ & $f a d \mathrm{NE} 35^{\mathrm{e}}$ \\
\hline $16: 0$ & $7.8 \pm 0.1$ & $8.0 \pm 0.1$ & $7.1 \pm 0.1$ & $6.4 \pm 0.1$ & $6.82 \pm 0.05$ \\
\hline 18:0 & $3.2 \pm 0.1$ & $4.1 \pm 0.1$ & $2.8 \pm 0.1$ & $2.59 \pm 0.02$ & $2.57 \pm 0.04$ \\
\hline $18: 1$ & $14.9 \pm 0.3$ & $31.9 \pm 0.5$ & $17.3 \pm 0.2$ & $37 \pm 2$ & $44.7 \pm 0.7$ \\
\hline $18: 2$ & $28.5 \pm 0.2$ & $15.2 \pm 0.3$ & $46.2 \pm 0.1$ & $24 \pm 2$ & $19.3 \pm 0.9$ \\
\hline $18: 3$ & $19.4 \pm 0.1$ & $10.1 \pm 0.3$ & ND & ND & ND \\
\hline $20: 0$ & $1.4 \pm 0.1$ & $1.84 \pm 0.08$ & $1.70 \pm 0.03$ & $1.47 \pm 0.05$ & $1.27 \pm 0.02$ \\
\hline $20: 1$ & $21.3 \pm 0.1$ & $24.6 \pm 0.1$ & $20.5 \pm 0.1$ & $22.7 \pm 0.3$ & $23.0 \pm 0.3$ \\
\hline $20: 2$ & $1.96 \pm 0.03$ & $0.93 \pm 0.03$ & $2.8 \pm 0.1$ & $1.1 \pm 0.2$ & $0.69 \pm 0.05$ \\
\hline $22: 1$ & $1.56 \pm 0.04$ & $1.38 \pm 0.03$ & $1.75 \pm 0.02$ & $1.40 \pm 0.07$ & $1.15 \pm 0.04$ \\
\hline Vernolic acid & ND & $1.80 \pm 0.05$ & ND & $2.9 \pm 0.4$ & $3.5 \pm 0.2$ \\
\hline 12-ероху-18:2 & ND & $0.3 \pm 0.1$ & ND & ND & ND \\
\hline
\end{tabular}

${ }^{a}$ Mean \pm standard deviation $(n=3-5)$

${ }^{b}$ Wild-type plants transformed with the control vector

${ }^{c}$ Wild-type plants transformed with the napin-epoxygenase construct

d fad3/fad7-1/fad 8 triple mutant

${ }^{\mathrm{e}}$ Two independent lines from the $\mathrm{fad} 3 / \mathrm{fad} 7-1 / \mathrm{fad} 8$ triple mutant transformed with the napin-epoxygenase construct $N D$ not detected 


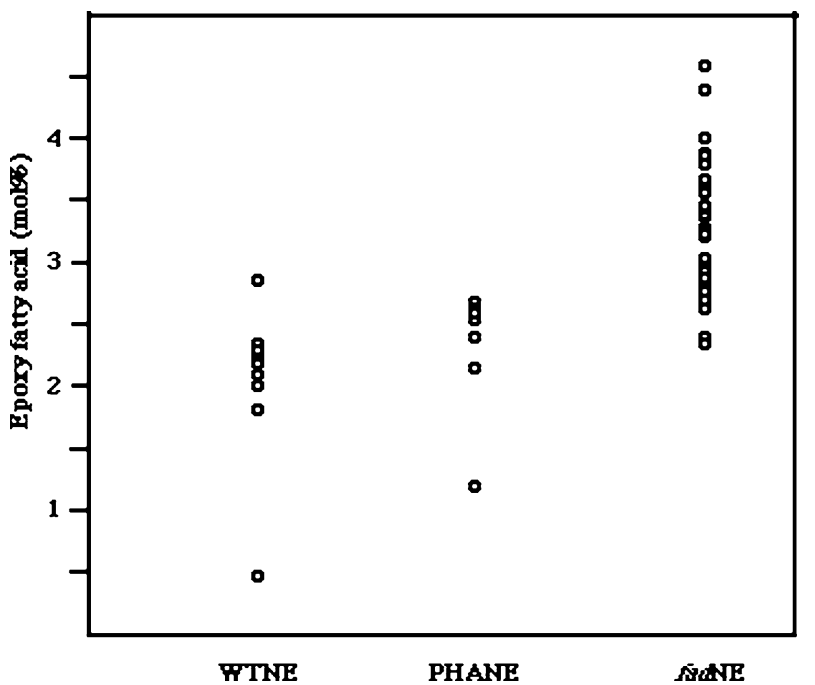

Fig. 1 Analysis of epoxy fatty acids produced in wild-type Aradidopsis thaliana and a mutant showing accumulation of linoleic acid. The napin-epoxygenase construct was transformed into either wild-type $A$. thaliana (WTNE), wild-type A. thaliana expressing a peroxisomal PHA synthase (PHANE) or the fad3/fad7-1/fad8 mutant $(f a d N E)$. The amount (in mol\%) of epoxy fatty acids (vernolic acid + 12-epoxy-18:2) measured in a pool of approximately 100 seeds obtained from each primary transgenic plant is indicated by a circle. Statistical analysis (ANOva) revealed no significant differences between WTNE and PHANE $(P=0.35)$, while highly significant differences were found between $\mathrm{fad} \mathrm{NE}$ and either WTNE or PHANE $(P<0.001)$

inhibits the activity of the native oleic acid $\Delta 12$-desaturase, thereby blocking the conversion of oleic acid to linoleic acid (Smith et al. 2003). Since linoleic acid is the substrate for the $C$. palaestina linoleic acid $\Delta 12$ epoxygenase, the reduced level of linoleic acid may be limiting the accumulation of epoxy fatty acids. It has been shown that overexpression of a $C$. palaestina $\Delta 12$-oleic acid desaturase along with the $C$. palaestina $\Delta 12$ epoxygenase in $A$. thaliana leads to increased levels of both linoleic acid and epoxy fatty acids (Singh et al. 2001). It is however unclear from this work if the increase in epoxy fatty acids was dependant solely on the increased level of linoleic acid or was rather the result of the coexpression of two enzymes from the native $C$. palaestina plant that could potentially interact to more efficiently convert oleic acid into vernolic acid. The presence of such functional multi-component enzyme association has been suggested to be important for the synthesis of unusual monoenoic acid fatty acids in transgenic Arabidopsis expressing a $\Delta 4$ 16:0-ACP desaturase (Suh et al. 2002).

In this investigation we used two independent methods of manipulating the level of available linoleic acid, and both led to increased synthesis of epoxy fatty acids in transgenic $A$. thaliana expressing the $C$. palaestina $\Delta 12$ epoxygenase. First, the growth of the transgenic plants in media containing linoleic acid showed a 2.8 -fold increase in the amount of vernolic acid, whereas the addition of the non-substrate linoelaidic acid led to a small decrease in vernolic acid. Second, expression of the $C$. palaestina $\Delta 12$-epoxygenase in the $\mathrm{fad} 3 / \mathrm{fad} 7-1 / \mathrm{fad} 8$ mutant, which is deficient in the synthesis of trienoic acid and shows a $60 \%$ increase in linoleic acid relative to the wild type, led to an approximately $55 \%$ increase in the amount of epoxy fatty acids. This level of increase in epoxy fatty acids is similar to the one observed in transgenic A. thaliana coexpressing the $C$. palaestina $\Delta 12$-epoxygenase and $\Delta 12$ desaturase (Singh et al. 2002). Together, these results indicate that higher levels of epoxy fatty acid production are mediated by the increased availability of the substrate linoleic acid.

The higher accumulation of the fatty acids $16: 1 \Delta 4$ and $18: 1 \Delta 6$ through the expression of the Coriandrum sativum $\Delta 4$ 16:0-ACP desaturase in the fabl mutant of A. thaliana, which is deficient in the elongation of 16:0ACP to 18:0-ACP, indicated that greater availability of the substrate 16:0-ACP to the $C$. sativum desaturase could also improve the accumulation of unusual monoenoic fatty acids (Suh et al. 2002). However, expression of the Thunbergia alata $\Delta 6$ 16:0- ACP desaturase in the same fabl mutant did not lead to significant changes in the accumulation of unusual monoenoic acids relative to the expression of the transgene in wild-type plants (Suh et al. 2002). Smith et al. (2003) recently examined the effects of manipulating the levels of oleic acid on the accumulation of hydroxy fatty acids in seeds of $A$. thaliana expressing the $R$. communis $\Delta 12$-oleic acid hydroxylase. Expression of the hydroxylase in a fael/fad 2 mutant accumulating $440 \%$ more oleic acid than a control line only led to a modest $30 \%$ increase in the average hydroxy fatty acid content and a $18 \%$ increase in the maximum hydroxy fatty acid content in the fael/fad 2 mutant compared to control (Smith et al. 2003). In contrast, the accumulation of epoxy fatty acids in seeds of $A$. thaliana expressing the $C$. palaestina $\Delta 12$-epoxygenase appears to be more responsive to the level of linoleic acid, since a modest increase of $60 \%$ in linoleic acid level was accompanied by a $55 \%$ increase in the average epoxy fatty acid content and a $60 \%$ increase in the maximum epoxy fatty acid content. Thus, improvement in the production of unusual fatty acids through an increased availability of the substrate may not apply to all transgenic plants and must, therefore, be evaluated case by case.

Despite the clear improvement in the accumulation of vernolic acid through the increased availability of linoleic acid, the absolute level of epoxy fatty acids in transgenic A. thaliana remained low (lower than $8 \mathrm{~mol} \%$ in vegetative tissues and lower than $5 \mathrm{~mol} \%$ in seeds) compared to that observed in the native $C$. palaestina plants (approximately $60 \mathrm{~mol} \%$ in seed lipids). Clearly, other factors are still limiting epoxy fatty acid accumulation in A. thaliana. Singh et al. (2001) reported the synthesis of epoxy fatty acids up to $6.2 \%$ in transgenic Arabidopsis expressing the C. palaestina linoleic acid $\Delta 12$-epoxygenase and up to $9.9 \%$ in plants co-expressing the $C$. palaestina linoleic acid $\Delta 12$-epoxygenase and $\Delta 12$-desaturase. The reasons for the lower amount of epoxy fatty acids in the transgenic plants obtained in this study are unknown but may be due to a combination of factors, including differences in the promoter $(0.5-\mathrm{kbp}$ vs. 
1.1-kbp napin promoter), binary vector (pBI121 vs. pCAMBIA 1300), ecotype (C24 vs. Columbia) and transformation method (hypocotyl or root transformation vs. floral dip).

The $C$. palaestina linoleic acid $\Delta 12$-epoxygenase acts on fatty acids present in phospholipids on the endoplasmic reticulum (Lee et al. 1998). The newly synthesized unusual fatty acids are then transferred from phospholipids to be mobilized into TAG via a number of potential routes, thereby implying the action of either specific phospholipases or acyltransferases, including the phosphatidylcholine:diacylglycerol acyltransferase (Dahlqvist et al. 2000; Ståhl et al. 1995). Potential other factors limiting epoxy fatty acids synthesis in transgenic plants could be the timing and absolute level of expression of the $\Delta 12$ epoxygenase, the availability of co-factors required for the epoxygenation reaction as well as the specificity of the enzymes involved in the stable incorporation of epoxy fatty acids into TAG. It is thus clear that in addition to increasing the availability of linoleic acid, further modification of plant metabolism through the use of mutants or the expression of additional transgenes will be required for high-level accumulation of epoxy fatty acids in transgenic plants.

Acknowledgements The authors are grateful to Nadine Erard for her technical assistance and Jerome Goudet for help with statistical analysis. This work was funded by a grant to YP from the OFES (grant number 99.0692) under the 5th Framework of the European Union (CONFAB project, GLRT-1999-00213). Contributions are also acknowledged from the University of Lausanne, the Herbette Foundation and the Canton de Vaud.

\section{References}

Badami RC, Patil KB (1981) Structure and occurrence of unusual fatty acids in minor seed oils. Progr Lipid Res 19:119-153

Broun P, Somerville C (1997) Accumulation of ricinoleic, lesquerolic, and densipolic acids in seeds of transgenic Arabidopsis plants that express a fatty acyl hydroxylase cDNA from castor bean. Plant Physiol 113:933-942

Broun P, Boddupalli S, Somerville C (1998) A bifunctional oleate 12-hydroxylase: desaturase from Lesquerella fendleri. Plant J 13: $201-210$

Budziszewski GJ, Croft KPC, Hildebrand DF (1996) Uses of biotechnology in modifying plant lipids. Lipids 31:557-569

Cahoon EB, Shanklin J, Ohlrogge JB (1992) Expression of a coriander desaturase results in petroselinic acid production in transgenic tobacco. Proc Natl Acad Sci USA 89:11184-11188

Cahoon EB, Carlson TJ, Ripp KG, Schweiger BJ, Cook GA, Hall SE, Kinney AJ (1999) Biosynthetic origin of conjugated double bonds: production of fatty acid components of high-value drying oils in transgenic soybean embryos. Proc Natl Acad Sci USA 96:12935-12940

Cahoon EB, Ripp KG, Hall SE, McGonigle B (2002) Transgenic production of epoxy fatty acids by expression of a cytochrome P450 enzyme from Euphorbia lagascae seed. Plant Physiol 128:615-624

Clough SJ, Bent AF (1998) Floral dip: a simplified method for Agrobacterium-mediated transformation of Arabidopsis thaliana. Plant J 6:735-743

Dahlqvist A, Stahl U, Lenman M, Banas A, Lee M, Sandager L, Ronne H, Stymne S (2000) Phospholipid:diacylglycerolacyltransferase: an enzyme that catalyzes the acyl-CoA independent formation of triacylglycerol in yeast and plants. Proc Natl Acad Sci USA 97:6487-6492
Eccleston VS, Cranmer AM, Voelker TA, Ohlrogge JB (1996) Medium-chain fatty acid biosynthesis and utilization in Brassica napus plants expressing lauroyl-acyl carrier protein thioesterase. Planta 198:46-53

Gleave P (1992) A versatile binary vector system with a T-DNA organisational structure conducive to efficient integration of cloned DNA into the plant genome. Plant Mol Biol 20:2031207

Jaworski J, Cahoon EB (2003) Industrial oils from transgenic plants. Curr Opin Plant Biol 6:178-184

Lee M, Lenman M, Banas A, Bafor M, Singh S, Schweizer M, Nilsson R, Liljenberg C, Dahlqvist A, Gummeson PO, Sjödahl S, Green A, Stymne S (1998) Identification of non-heme diiron proteins that catalyze triple bond and epoxy group formation. Science 280:915-918

Loo FJ van de, Fox BG, Somerville C (1993) Unusual fatty acids. In: Moore TS (ed) Lipid metabolism in plants. CRC Press, Baton Rota

Loo FJ van de, Broun P, Turner S, Somerville C (1995) An oleate 12-hydroxylase from Ricinus communis L. is a fatty acyl desaturase homolog. Proc Natl Acad Sci USA 92:6743-6747

McConn M, Browse J (1996) The critical requirement for linolenic acid is pollen development, not photosynthesis, in an Arabidopsis mutant. Plant Cell 8:403-416

Mittendorf V, Bongcam V, Allenbach L, Coullerez G, Martini N, Poirier, Y (1999) Polyhydroxyalkanoate synthesis in transgenic plants as a new tool to study carbon flow through $\beta$-oxidation. Plant J 20:45-55

Moire L, Rezzonico E, Goepfert S, Poirier Y (2004) Impact of unusual fatty acid synthesis on futile cycling through $\beta$ oxidation and on gene expression in transgenic plants. Plant Physiol 134:432-442

Murashige T, Skoog F (1962) A revised medium for rapid growth and bioassays with tobacco tissue cultures. Physiol Plant $15: 473-479$

Poirier Y (2002) Poylhydroxyalkanoate synthesis in plants as a tool for biotechnology and basic studies of lipid metabolism. Progr Lipid Res 41:131-155

Poirier Y, Ventre G, Calelari D (1999) Increased flow of fatty acids toward $\beta$-oxidation in developing seeds of Arabidopsis deficient in diacylglycerol acyltransferase activity or synthesizing medium-chain-length fatty acids. Plant Physiol 121:13591366

Singh S, Thomaeus S, Lee M, Stymne S, Green A (2001) Transgenic expression of a $\Delta 12$-epoxygenase gene in Arabidopsis seeds inhibits accumulation of linoleic acid. Planta 212:872-879

Smith MA, Moon H, Chowrira G, Kunst L (2003) Heterologous expression of a fatty acid hydroxylase gene in developing seeds of Arabidopsis thaliana. Planta 217:507-516

Ståhl U, Banas A, Stymne S (1995) Plant microsomal phospholipid acyl hydrolases have selectivities for uncommon fatty acids. Plant Physiol 107:953-962

Stumpf PK, Weber N (1977) Uptake and metabolism of fatty acids by soybean suspension cells. Lipids 12:120-124

Suh MC, Schultz DJ, Ohlrogge JB (2002) What limits production of unusual monoenoic fatty acids in transgenic plants. Planta 215:584-595

Terzaghi WB (1986a) A system for manipulating the membrane fatty acid composition of soybean cell cultures by adding tween-fatty acid esters to their growth medium. Plant Physiol 82:771-779

Terzaghi WB (1986b) Metabolism of tween-fatty acid esters by cultured soybean cells. Plant Physiol 82:780-786

Terzaghi WB (1989) Manipulating membrane fatty acid compositions of whole plants with tween-fatty acid esters. Plant Physiol 91:203-212

Voelker T, Kinney AJ (2001) Variations in the biosynthesis of seed storage lipids. Annu Rev Plant Physiol Plant Mol Biol 52:335361 